\title{
Symptom Triggered Therapy in Delirium Tremens
}

\author{
Lt Col J Prakash*, Col R Setlur ${ }^{+}$, Lt Col RK Saini ${ }^{\#}$
}

MJAFI 2010; 66 : 180-181

Key Words : Symptom triggered therapy; Delirium tremens

\section{Introduction}

$\mathrm{A}$ lcohol withdrawal due to inadvertent abstinence in alcohol dependent patients is a significant problem. These patients frequently suffer from severe withdrawal or delirium tremens, which requires intensive management. Delirium tremens has a mortality rate of $1-5 \%$ [1]. Prevention of alcohol withdrawal syndrome has been shown to improve morbidity and mortality and shorten hospital stay [2]. Benzodiazepines have been shown to be safe and effective for preventing or treating seizures and delirium and are the preferred agents for treating alcohol withdrawal syndrome [1,3]. A symptomtriggered approach to dosing benzodiazepenes may have significant advantages over a fixed schedule therapeutic approach. These include the ability to individualise treatment, reduction in treatment duration and reduced occurrence of delirium tremens $[1,4]$. Previous studies have shown equally efficacious results with a symptom triggered approach [5]. There is, however, a requirement of suitably trained staff [1].

We report a case of alcohol dependence, which was admitted to the medical ward for dyspepsia but progressed to develop florid delirium tremens. He was initially managed with standard fixed schedule therapy but when this proved ineffective, he was switched to symptom-triggered therapy. An intensive care approach with continuous monitoring and timely use of therapeutic interventions lead to a successful outcome in a potentially life threatening situation.

\section{Case Report}

A 46 year old exserviceman presented to a hospital with recurrent epigastric pain and nausea, which had not improved despite prolonged outpatient treatment. Initially on admission he gave a history of alcohol abuse to the tune of 60-120 $\mathrm{ml}$ of rum on a daily basis. He claimed to have been abstinent for the past 3-4 weeks. On examination, the patient had epigastric tenderness. Vital parameters were within normal limits.
Relevant investigations revealed slightly raised serum gamma glutamine transferase (SGGT) to 67 IU/1 and hepatomegaly with grade I fatty changes on ultrasonography (USG) of abdomen. Electrocardiograph (ECG) and other biochemical parameters were normal. He was started on symptomatic management with antacids and prokinetics. By evening, i.e. after around 10 hours of the admission he was noticed to be talking irrelevantly, confused, wandering aimlessly, neglecting self-care and not recognizing people. The symptomatology rapidly progressed to extreme agitation. A psychiatric consultation was sought at this juncture. He had tachycardia (pulse 120/min), elevated blood pressure (BP 146/98), tremulousness, hyperhidrosis, psychomotor agitation, irrelevant speech, disorientation, confusion, paranoid ideation and deranged biodrives. Further history elicited from the family members revealed that he consumed around $750 \mathrm{ml}$ of alcohol per day for last 15 years and had not stopped until the night before admission. Management was started on the line of alcohol dependence syndrome and alcohol withdrawal syndrome with delirium tremens. He was continued on forced abstinence, started on detoxification with oral chlordiazepoxide (upto $120 \mathrm{mg}$ /day in divided dosage), injectable haloperidol for agitation control and other supportive measures. The injectable haloperidol was started in the dose of $5 \mathrm{mg}$ to start with and in the absence of any appreciable response the same was increased to $10 \mathrm{mg}$ intramuscular (IM). A total of three such injections were given to the patient after every 2-3 hours of observation. However no appreciable improvement was seen clinically even after 12 hours of observation. He developed low grade fever the next morning. Periodic assessment did not reveal any other pathological process. Contrast enhanced computed tomograph (CECT) cranium was within normal limits. Analysis of blood creatinine phosphokinase (CPK) showed rising titres. In view of poor response to adequate dosage of injectable neuroleptics, continued psychomotor agitation, autonomic disturbances, rhabdomyolysis and possibility of a neuroleptic malignant syndrome with further neuroleptics, revision of treatment was considered. Symptom triggered management of the patient with intermittent bolus of injectable midazolam was initiated. He was shifted to the intensive care unit (ICU)

${ }^{*}$ Reader (Department of Psychiatry), AFMC, Pune-40. ${ }^{+}$Senior Advisor (Anaesthesia \& Critical Care), ${ }^{\#}$ Classified Specialist (Psychiatry), 167 Military Hospital, C/o 56 APO.

Received : 28.07.09; Accepted : 08.02.10

E-mail : drjyotiprakashpsy@yahoo.com 
Table 1

Total amount of Midazolam used per day in ICU

$\begin{array}{llc}\text { Srl No } & \text { Day in ICU } & \text { Total midazolam used/day } \\ 1 & \text { Day } 1 & 23 \mathrm{mg} \\ 2 & \text { Day } 2 & 18 \mathrm{mg} \\ 3 & \text { Day } 3 & 9 \mathrm{mg}\end{array}$

for effective monitoring of cardio-respiratory status. He was sedated with a bolus dose of $5 \mathrm{mg}$ intravenous (IV) midazolam and started on IV dextrose saline to prevent hypoglycaemia and dehydration. Recurrence of agitation or positive glabellar tap was taken as an indication for repeat bolus of midazolam. By the second day he had become afebrile, his tachycardia subsided, blood pressure normalized and sweating was much less. His CPK level came down to normal. Total dose of midazolam used per day is as per Table 1 . On $3^{\text {rd }}$ day he was found well-oriented and talking relevantly when he woke up from the effect of midazolam. He had minimal withdrawal features and showed no agitation. After three days in ICU he was shifted to medical ward on oral chlordiazepoxide $(50 \mathrm{mg} /$ day), which was tapered off in a week. He was started on the anticraving agent, acamprosate, on seventh day. After two weeks in the hospital he was discharged on acamprosate, with follow up instructions.

\section{Discussion}

This 46 year old exserviceman reported primarily with complaints of dyspepsia. He manifested with withdrawal features due to abstinence imposed involuntarily by hospital admission. He had severe withdrawal features with delirium, which was predictable in view of the duration and quantity of his alcohol consumption [1,3]. As his withdrawal delirium and agitation was not controlled by fixed schedule detoxification, symptom triggered therapy was started. He was shifted to the ICU and kept sedated through the period of delirium. He recovered significantly and faster with this regime than the standard regime in practice. These findings are akin to existing literature, which shows a reduction in benzodiazepines requirement and duration of treatment [6]. Although benzodiazepine use and duration of treatment did not differ significantly in Mayo clinic protocol, researchers advocated symptom triggered therapy as effective treatment in alcohol withdrawal syndrome as well as for reducing the risk of delirium tremens while emphasizing further studies to appreciate other benefits [4]. Withdrawal features abated in a week and he was discharged in two weeks. The findings are similar to other researchers [6].

To conclude, symptom triggered therapy was found extremely efficacious when a fixed dose approach was ineffective. Increased staffing requirements and intensive monitoring of this approach seems justified by the successful outcome. It would be worthwhile to carry out a full-fledged trial to see applicability of this approach in service hospitals.

\section{Conflicts of Interest}

None identified

\section{References}

1. Bayard M, McIntyre J, Hill KR, Woodside J. Alcohol Withdrawal Syndrome. Am Fam Physician 2004; 69:1443-50.

2. Dissanaike S, Halldorsson A, Frezza EE, Griswold JJ. An ethanol protocol to prevent alcohol withdrawal syndrome. Am Coll Surg 2006 ; 203: 186-91.

3. Alcohol withdrawal syndrome: how to predict, prevent, diagnose and treat it. Prescrire Int 2007; 16: 24-31.

4. Jaeger TM, Lohr RH, Pankratz VS. Symptom-triggered therapy for alcohol withdrawal syndrome in medical inpatients. Mayo Clin Proc 2001; 76: 695-701.

5. Daeppen JB, Gache P, Landry U, Sekera E, Schweizer V, Gloor $\mathrm{S}$, et al. Symptom-Triggered vs. Fixed-Schedule Doses of Benzodiazepine for Alcohol Withdrawal: A Randomised Treatment Trial. Arch Intern Med 2002; 162:1117-21.

6. De Carolis DD, Rice KL, Ho L, Willenbring ML, Cassaro S. Symptom-driven lorazepam protocol for treatment of severe alcohol withdrawal delirium in the intensive care unit. Pharmacotherapy 2007; 27: 510-8. 